\title{
Validation of a clinical PET scanner using Monte Carlo simulation code: MCNP5
}

\begin{abstract}
This paper presents a realistic PET scanner simulation using Monte Carlo code, version MCNP5. The objective of our study was to verify the code used for this simulation by comparing the results obtained from the simulation with those obtained from the measurement did on a real PET scanner. This study will provide a basic benchmark for our further study on PET imaging. We modeled the scanner based on the physical specification of Siemens Biograph True Point PET scanner. We recorded the generated list-mode data which contains all the information needed to model the PET processes for instance coincidence photon detection. To account for the statistical fluctuations occur in the detector and photo multiplier tube, a Gaussian energy blurring model was applied to the energy deposited in the detector. The scatter and attenuation correction to correct the effect of scattered and attenuated events also took into account in this study. All of these post-simulation processes were done using a program developed using mat lab. To validate the simulation, the simulated and measured energy and spatial resolution were compared. We have successfully modeled a PET system based on MCNP5. We also verified that this simulation result in a good agreement data with the real imaging.
\end{abstract}

Keyword: Validation; Monte Carlo; PET 